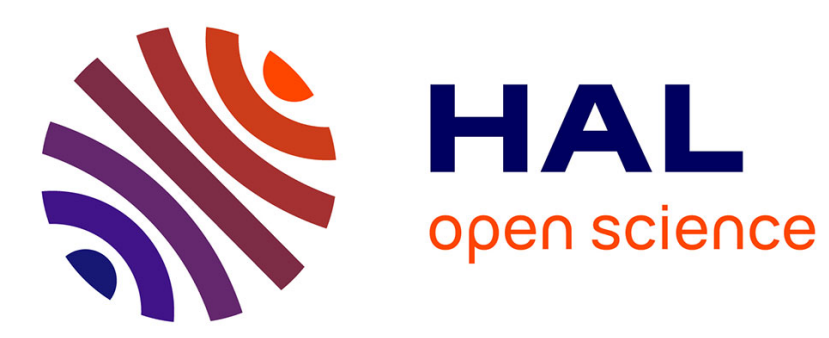

\title{
Monte Carlo static and dynamic simulations of a three-dimensional Ising critical model
}

Frédéric Livet

\section{To cite this version:}

Frédéric Livet. Monte Carlo static and dynamic simulations of a three-dimensional Ising critical model. Physical Review E , 2020, 101 (2), 10.1103/PhysRevE.101.022131 . hal-02941259

HAL Id: hal-02941259

https://hal.univ-grenoble-alpes.fr/hal-02941259

Submitted on 16 Sep 2020

HAL is a multi-disciplinary open access archive for the deposit and dissemination of scientific research documents, whether they are published or not. The documents may come from teaching and research institutions in France or abroad, or from public or private research centers.
L'archive ouverte pluridisciplinaire HAL, est destinée au dépôt et à la diffusion de documents scientifiques de niveau recherche, publiés ou non, émanant des établissements d'enseignement et de recherche français ou étrangers, des laboratoires publics ou privés. 


\title{
Monte Carlo static and dynamic simulations of a three-dimensional Ising critical model
}

\author{
Frédéric Livet $\oplus^{*}$ \\ Université Grenoble Alpes, CNRS, Grenoble-INP, SIMaP, F-38000 Grenoble, France
}

(Received 1 December 2019; accepted 10 February 2020; published 27 February 2020)

\begin{abstract}
The critical dynamics of "model A" of Hohenberg and Halperin has been studied by the Monte Carlo method. Simulations have been carried out in the three-dimensional (3d) simple cubic Ising model for lattices of sizes $L=16$ to $L=512$. Using Wolff's cluster algorithm, the critical temperature is precisely found as $\beta_{c}=0.22165468(5)$. By Fourier transform of the lattice configurations, the critical scattering intensities $I(\vec{q})$ can be obtained. After circular averaging, the static simulations with $L=256$ and $L=512$ provide an estimate of the critical exponent $\gamma / v=2-\eta=1.9640(5)$. The $|\vec{q}|$-dependent distribution of $I(\vec{q})$ showed an exponential distribution, corresponding to a Gaussian distribution of the scattering amplitudes for a large $q$ domain. The time-dependent intensities were then used for the study of the critical dynamics of $3 \mathrm{~d}$ lattices at the critical point. To simulate results of an x-ray photon correlation spectroscopy experiment, the time-dependent correlation function of the intensities was studied for each $|\vec{q}|$-value. In the $q$ region where $I(\vec{q})$ had an exponential distribution, the time correlations can be fit to a stretched exponential, where the exponent $\mu=\gamma / \nu z \simeq 0.975$ provides an estimate of the dynamic exponent $z$. This corresponds to $z=2.0145$, in agreement with the observed variations of the characteristic fluctuation time of the intensity: $\tau(q) \propto q^{-z}$, which gives $z=2.015$. These results agree with the $\epsilon$ expansion of field-theoretical methods (2.017). In this paper, the need to take account of the anomalous time behavior $(\mu<1)$ in the dynamics is exemplified. This dynamics reflects a nonlinear time behavior of model A, and its large time extension is discussed in detail.
\end{abstract}

DOI: 10.1103/PhysRevE.101.022131

\section{INTRODUCTION}

\section{A. Scaling}

The well-established theory of critical systems belonging to the class of three-dimensional (3d) Ising systems stresses the importance of the fluctuations in the vicinity of the critical point. These are studied in scattering experiments (neutrons, X-rays, electrons), and the results for $T>T_{c}$ can be connected to a unique fluctuation length $\xi: \xi \propto\left|T-T_{c}\right|^{-v}$. The "model A" dynamics was summarized in Ref. [1]. In this model, the local order parameter $\phi(\vec{r})$ is not conserved and fluctuates dynamically. The corresponding dynamic Ising model is based on a local spin flip and the collective dynamics at a given scale $\Delta L \gg 1$ is studied if unity is the lattice constant.

For a system in the vicinity of the critical temperature $T_{c}$, i.e., for a given $\xi$, the dynamic scaling can be written from the first-order correlations of the Fourier transform $A(\vec{q}, t)$ of the magnetization $m(\vec{r}, t)$ :

$$
S(\vec{q}, t)=\left\langle A\left(\vec{q}, t+t^{\prime}\right) A^{\star}(\vec{q}, t)\right\rangle_{t^{\prime}} / N,
$$

where $N=L^{3}$ is the number of simple cubic sites and $m(\vec{r})=$ \pm 1 . In the case of a second-order transition with a monodimensional order parameter $S(\vec{q})$ is isotropic on average and, introducing explicitly $\xi$, the dynamic scaling hypothesis [2] can be written as

$$
S(0, \xi, t)=b^{\gamma / v} S\left(q b, \xi b^{-1}, t b^{-z}\right),
$$

\footnotetext{
*frederic.livet@grenoble-inp.fr
}

where $\gamma$ and $v$ have the classical meaning in critical systems (some estimates are collected in Ref. [3]). Here, their ratio is also written: $\gamma / v=2-\eta \simeq 1$.96. The correlations can be observed from the relaxation of $S(q, t)$, and a characteristic time $\tau$ dependent on $q$ and $\xi$ can be introduced. From Eq. (2) one can obtain the limiting behavior for various cases of interest by the appropriate choice of $b$.

To get an estimate of the characteristic time $\tau$, one chooses $b=\xi$, which gives $(q=0)$

$$
S(q, \xi, 0)=\xi^{\gamma / \nu} S\left(0,1, t \xi^{-z}\right),
$$

which means $\tau \propto \xi^{z}$ and, for $t=0\left(t \ll \xi^{z}\right)$, the static scattering intensity is written

$$
S(q, \xi, 0)=\xi^{\gamma / v} S(q \xi, 1,0)
$$

Writing $b=t^{-z}$ and $\xi=\infty$ (i.e., $q \gg \xi^{-1}$ ) yields

$$
S(q, \infty, t)=q^{-\gamma / v} S\left(1, \infty, t q^{z}\right)=q^{-\gamma / v} \Phi\left(x=t q^{z}\right),
$$

which means $\tau \propto q^{-z}$.

In Eq. (5), the shape of $\Phi\left(x=t q^{z}\right)$ for $t \ll \xi^{z}$ is not known, but it can be shown that its Fourier transform $G(q, \omega)$ has a singular behavior $\left(\omega \gg \xi^{-z}\right)[4,5]$ :

$$
G(q, \omega) \propto 1 / \omega^{1+\gamma / v z}
$$

This exponent (called in this paper $\mu=\gamma / \nu z$ ) was introduced in the two-dimensional (2d) Ising system [6] from the study of the time Fourier transform of $S(q=0, t)$. This means that the 


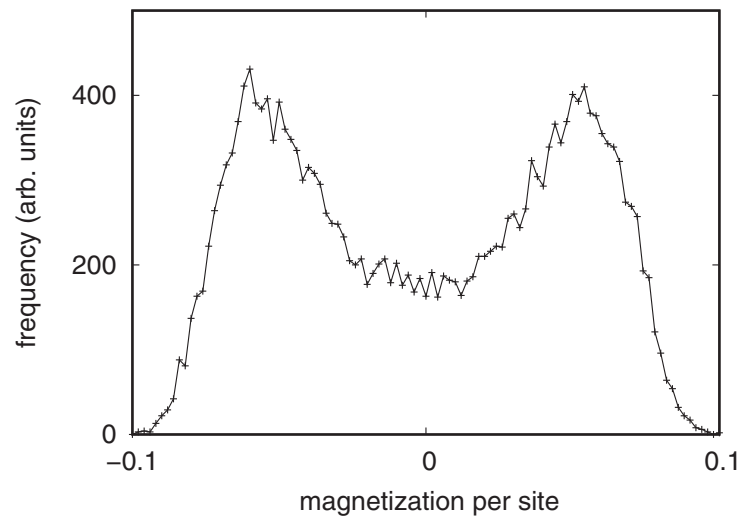

FIG. 1. The distribution of the per atom lattice magnetization $\operatorname{\Sigma m}(\vec{r}) / N$ for $L=512$ obtained from 22000 independent samples at $\beta=0.22165469$.

time behavior of this function $\Phi$ contains a $t^{\mu}$ singular time dependence.

To first order, this singularity can be written

$$
S(q, \infty, t) \simeq S(q, \infty, 0)-\left(t / t_{0}\right)^{\mu} .
$$

This time dependence is discussed here from a study of the dynamics of the $3 \mathrm{~d}$ Ising model, and Eq. (7) is interpreted as the first term of a stretched exponential behavior of the function $\Phi(q, t)$.

The dynamic scaling exponent $z$ has been estimated by field-theoretical methods $[2,7,8]$; the best value seems to be $z=2.017$ [9], and the results of numerous Monte Carlo simulations are in the range $z=2.03-2.09$ [10-13].

\section{B. An experiment}

This behavior has been studied in the model system $\mathrm{AuAgZn}{ }_{2}[14,15]$. For this dynamical study, the $\mathrm{x}$-ray photon correlation spectroscopy (XPCS) technique was used at the European Synchrotron Radiation Facility (ESRF) ID10 beamline. The time dependence of the coherent x-ray scattering intensity $I(\vec{q}, t)$ was measured. The time-correlation of this intensity can be written as

$$
G(\vec{q}, t)=\frac{\left\langle I\left(\vec{q}, t^{\prime}\right) I\left(\vec{q}, t+t^{\prime}\right)\right\rangle_{t^{\prime}}}{\left\langle I\left(\vec{q}, t^{\prime}\right)\right\rangle_{t^{\prime}}^{2}},
$$

and this normalized correlation, after angular averaging, is written

$$
G(q, t)=1+K g^{(2)}(q, t) .
$$

In Eq. (9), $K$ is the speckle contrast (the square of the "visibility") and we define $g^{(2)}$, the variable part of the second-order normalized correlation function (see definitions in Ref. [16]). In the case of the XPCS experiment [15], the size of the coherently illuminated sample was of the order of $20 \mu \mathrm{m}$ and the maximum fluctuation length $\xi$ was less than $\simeq 0.1 \mu \mathrm{m}$. The large number of fluctuations in the irradiated sample ensures that the central limit theorem holds and that the distribution of the scattering amplitude had a Gaussian

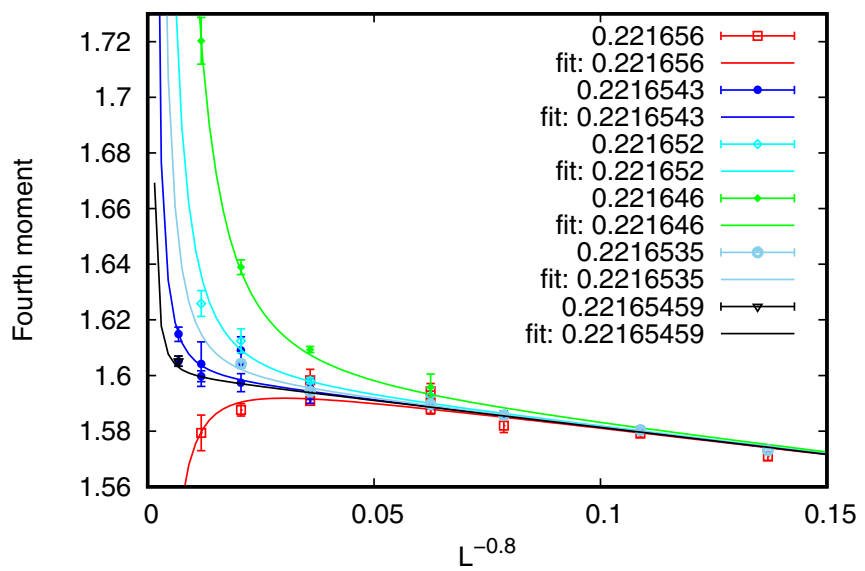

FIG. 2. The variations of the fourth moments $g^{(4)}$ vs $L^{-0.8}$ and $\beta$ and the calculated results of the fit using Eq. (12) with $\beta_{c}=$ 0.22165468 .

distribution. In this case, the Siegert relation [17,18] holds:

$$
g^{(2)}(q, t)=\left|g^{(1)}(q, t)\right|^{2}=|S(q, t) / S(q, 0)|^{2} .
$$

This experiment on the $\mathrm{AuAgZn} \mathrm{n}_{2}$ system was devoted to the observation of the critical slowing-down. The essential result was in the experimental estimates of $\tau(q, T)$; from these estimates, the value $z=1.96(11)$ was obtained. This result of a poor precision should be significantly improved with the new ESRF EBS source in progress [19].

The aim of this paper is to discuss how to estimate $z$ from Monte Carlo simulations in a finite system of size $L$ at $T_{c}$ and to try to improve the description of the dynamics of the fluctuations in a critical system.

\section{MONTE CARLO TECHNIQUE}

The classical Monte Carlo (MC) algorithm is a microcanonical probability of a spin flip, expressed from the energy change $\Delta E$, and, in order to simulate the time with a

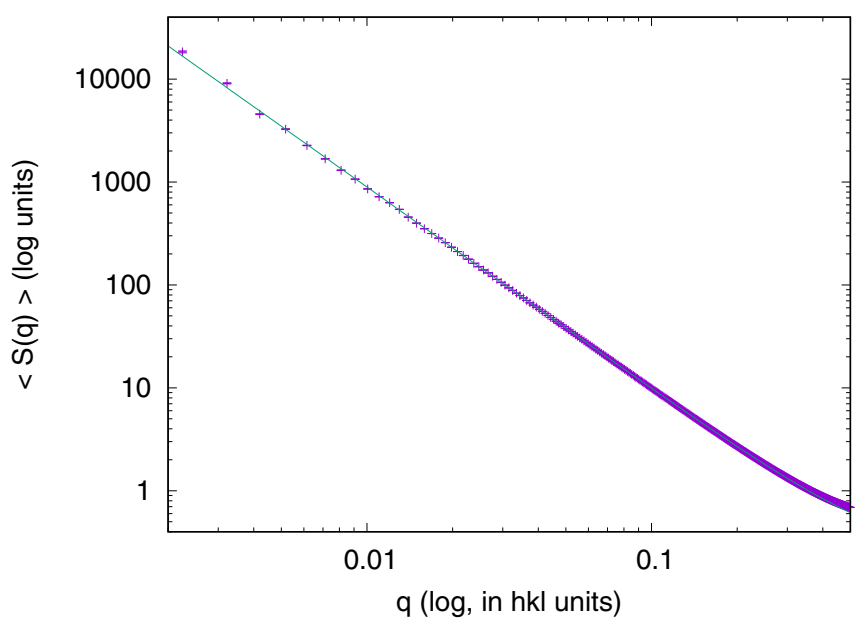

FIG. 3. Simulation of the critical intensity $S(q)$ for $L=512$ and result of the fit to Eq. (14) with $a_{0}=0.197, a_{1}=0.106$, and $a_{2}=$ 1.9627. 

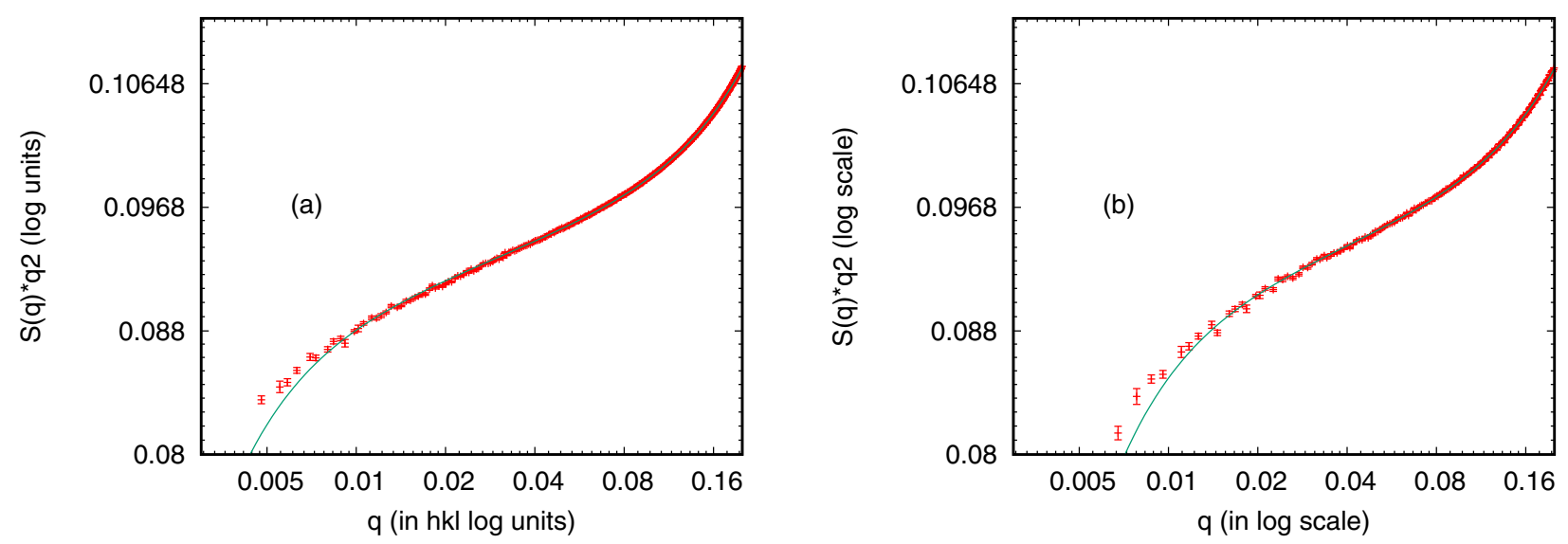

FIG. 4. $q^{2} I(q)$ for (a) $L=512$ and (b) $L=256$. The curves correspond to fits with Eq. (15).

"daemond" moving across the simple cubic lattice, the flip probability is fixed to:

$$
p_{\text {flip }}=\exp (-\beta \Delta E) /[1+\exp (-\beta \Delta E)],
$$

where $\beta$ is the classical inverse temperature in the Ising model. Sites are randomly explored and the time unit is the Monte Carlo step (MCS), i.e., an exploration of $L^{3}$ sites. For long enough time, this method gives a good simulation of the collective movement of the fluctuations in the sample. Upon carrying out simulations around the critical temperature, the MC method is faced with the critical slowing-down: the computation time in a finite system of size $L$ increases like $L^{z}$, which has to be multiplied by the number of elementary processes [Eq. (11)] $L^{3}$ for one MCS. If large systems are to be studied at the critical temperature, only a very small number of independent states can be examined, and the critical equilibrium state is even difficult to guarantee. For this reason, some simulations are carried out with large $L$, and the dynamics is studied by quenching to the critical point $\beta_{c}$ from disorder or by studying disordering from a fully ordered state, as in Ref. [20].

In this paper, a combination of classical kinetics and of Wolff's [21] algorithm is used for system equilibration and, for a static study, Wolff's algorithm provides a set of independent critical states in a reasonable computation time, as discussed in Ref. [22].

\section{A. Critical temperature}

The classical method for the identification of the critical point is the study of the size and temperature variation of the fourth-order moment of the distribution of the global magnetization [23]: $g^{(4)}=\left\langle m^{4}\right\rangle /\left\langle m^{2}\right\rangle^{2}$ which in the vicinity of $\beta_{c}$ is written

$$
g^{(4)}(L, \beta)=g_{0}^{(4)}+a_{0} L^{-\omega}+\left[\partial g^{(4)}\left(L, \beta_{c}\right) / \partial \beta\right]\left(\beta-\beta_{c}\right) .
$$

In the classical theory of the critical point, the distribution of the moments of the lattice is not Gaussian, as shown in Fig. 1, and Fig. 2 shows the plot of $g^{(4)}$ for various values of $L$ and $\beta$.

In Eq. (12), it is assumed that the main $L$ dependence is the $L^{-\omega}$ correction to the scaling laws [24], with $\omega=0.8$. From the results summarized in Fig. 2, the best fit yields an estimate of $\beta_{c}=0.22165468(5)$. This result is in agreement with our previous estimate $\left(\beta_{c}=0.2216544(10)\right.$ [22]) and with the two most recent estimates $[25,26]$ of $\beta_{c}=0.221654631(8)$
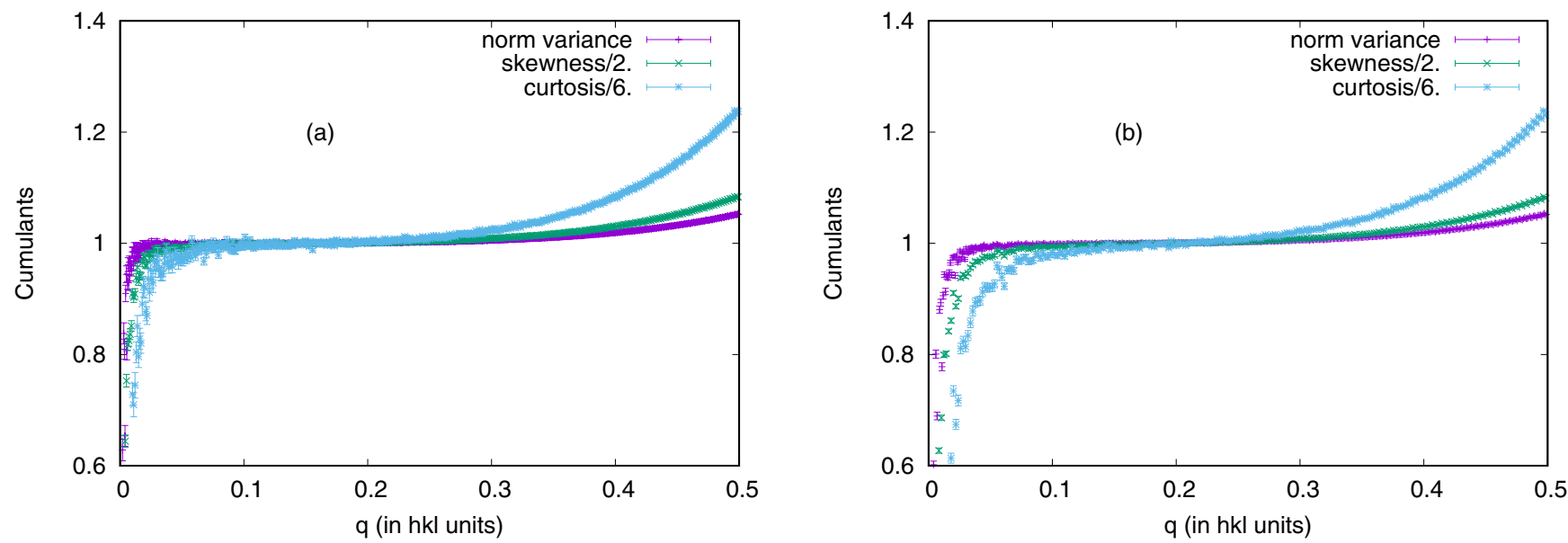

FIG. 5. The cumulants of the distribution of the intensities $I(q)$ clearly show the Gaussian distributions for (a) $L=512$ and (b) $L=256$. 


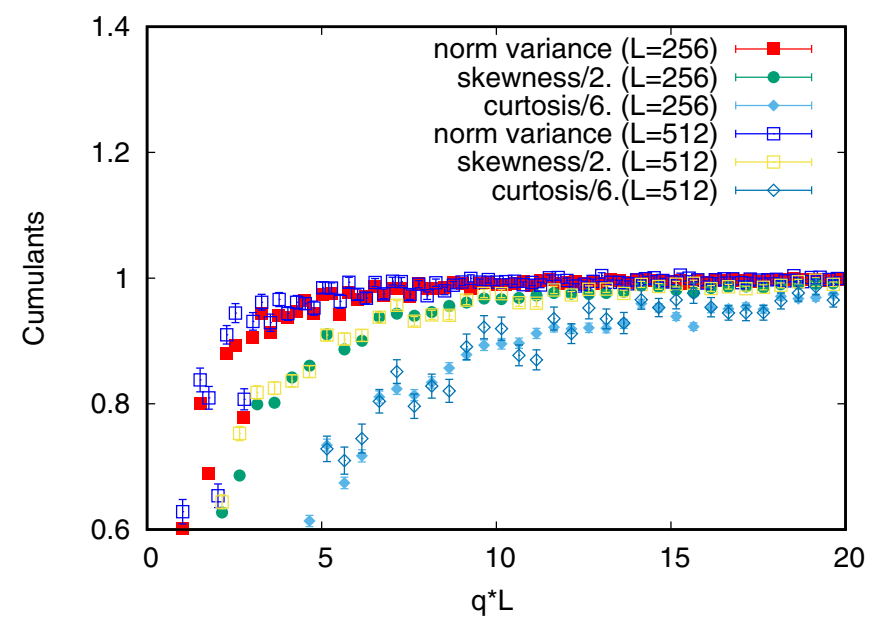

FIG. 6. Detail of the cumulants showing their $q L$ dependence and the Gaussian distribution for $q L>10-15$.

and $\beta_{c}=0.221654626(5)$. The values $g_{0}^{(4)}=1.6006(6)$ and $a_{0}=-193(7)$ are significantly different from our previous result in Ref. [22].

After this estimate of the critical temperature, all simulations reported here were carried out at $\beta=0.22165469$.

\section{B. Static critical scattering}

From a simulated configuration $m(\vec{r}, j)$, the per site critical scattering $I(\vec{q}, j)$ is calculated by a Fourier transform $\left(N=L^{3}\right)$ :

$$
I(\vec{q}, j)=\left|\sum m(\vec{r}, j) \exp (2 \pi \vec{r} \vec{q})\right|^{2} / N=|A(\vec{q}, j)|^{2} / N
$$

$I(\vec{q}, j)$ corresponds to the instantaneous intensity in an XPCS measurement, with a contrast $K=1$, and it has a "speckle" pattern, depending on the configuration $j$. In this paper, the $q$ values will be given in " $h k l$ " units, so that the position of the first Bragg peak is $q=1 / a=1$ if the lattice parameter $a$ is unity.

With the intensity defined in Eq. (13), one has in a Brillouin zone (BZ): $\iiint_{Z B} I(\vec{q}) d^{3} q \simeq 1$.

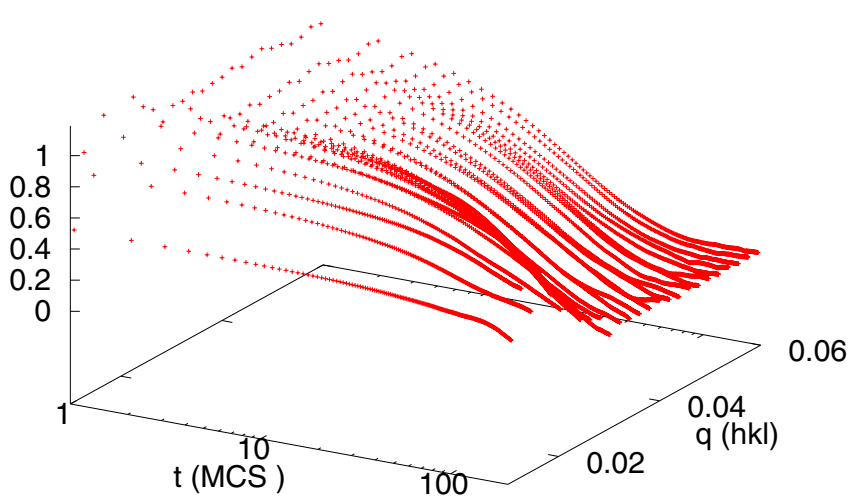

FIG. 7. Correlations $g^{(2)}(q, t)$ plotted vs $\log ($ time $)$ and $q$ for $L=$ 256 and 200 MCS.

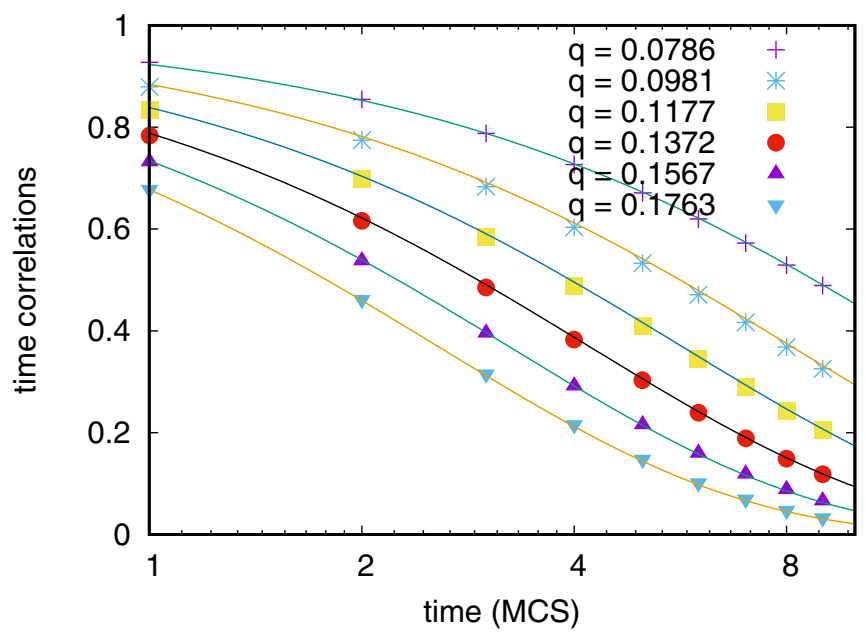

FIG. 8. Normalized correlation $g^{(2)}$ plotted vs $\log ($ time $)$ for short time $(t<10 \mathrm{MCS})$ and for a small set of $q$ values of the simulations: $L=512$, the fits (obtained from all $120 q$ values) are in excellent agreement with a simple exponential.

In Fig. 3 the intensities plotted are obtained by averaging over the $3 \mathrm{~d}$ reciprocal volume: each $I(q)$ value corresponds to an average of 1000 independent configurations with $\delta q=$ 0.001 for $L=512$ lattices. The set of measured intensity provides a good estimate of the statistical errors, and a good quality fit is obtained from $q=0.03$ to $q=0.17$ with the equation

$$
I(q)=a_{0}+a_{1} / q^{a_{2}},
$$

where $a_{2}=\gamma / v=1.9627(20)$. To focus on the small $q$ values, Fig. 4 shows a plot of $q^{2} I(q)$ vs $q$. The rounding for $q<0.02(L=512)$ and for $q<0.04(L=256)$ is connected to the finite size of the lattice and is observed for $q<10 / L$.

To take account of this rounding, a more complex fit with Eq. (15) was carried out:

$$
I(q)=a_{0}+a_{1} /\left(a_{3} q^{a_{2}}+1\right) .
$$

This equation is a small modification to the Lorentzian meanfield result, and better estimates of the critical exponent were obtained from this equation in the range $0.005 q$ to $-0.16 q$ $(L=512)$ and in the range $0.01 q$ to $0.16 q$ for $L=256$. Results were $\gamma / v=1.9643(2)(L=512)$ and $\gamma / v=1.9637(4)$

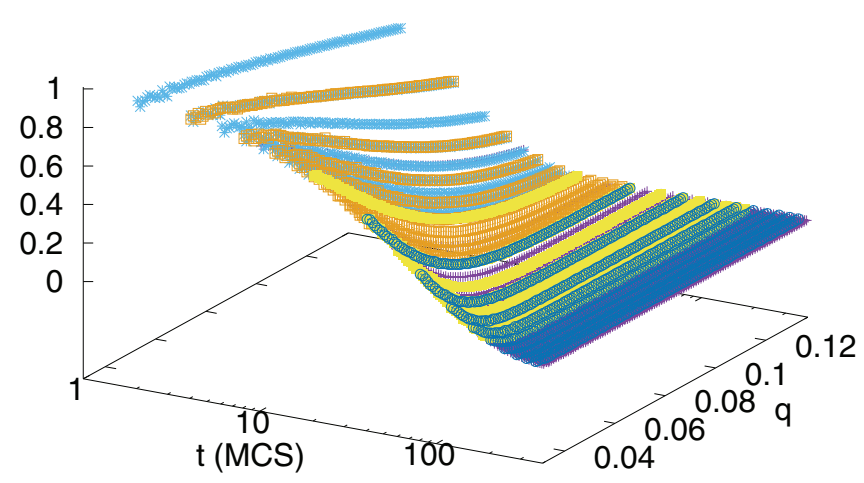

FIG. 9. $g^{(2)}(q, t)$ plotted vs $\log ($ time $)$ and $q$ for $L=512$ and various sampling periodicities (1, 2, 4, 10, 20, $40 \mathrm{MCS})$. 

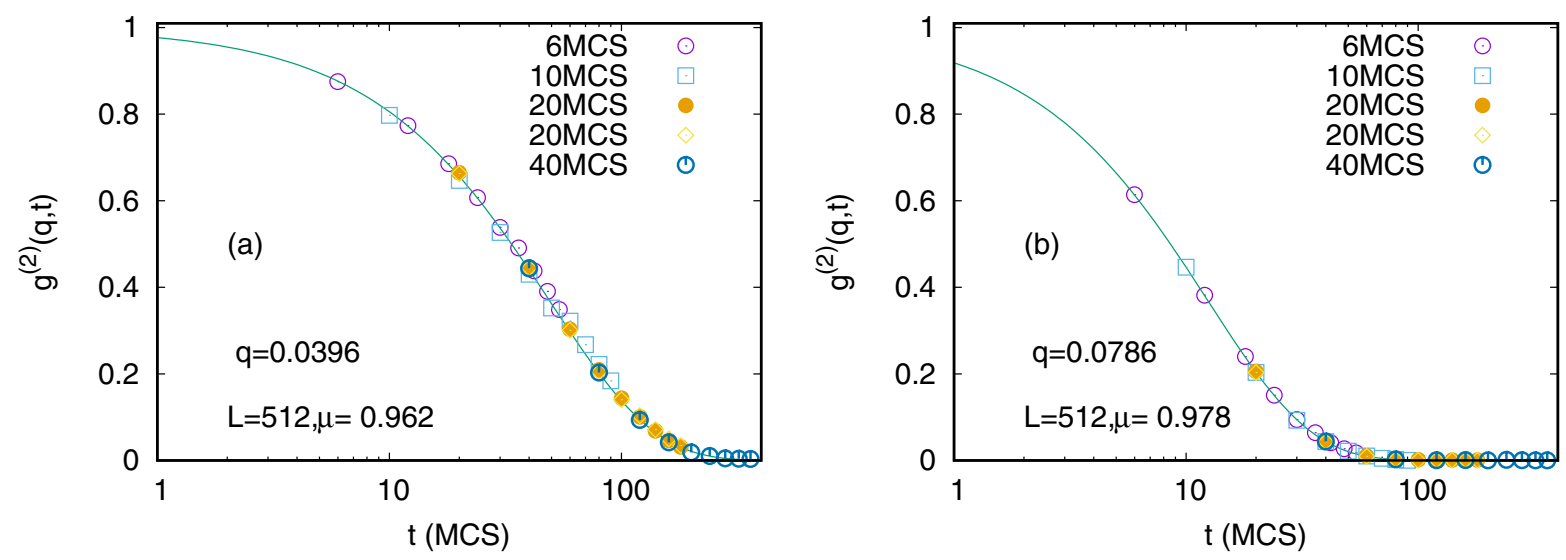
fits.

FIG. 10. Normalized correlation $g^{(2)}(q, t)$ plotted vs $\log ($ time) for (a) $q=0.0396$ and (b) $q=0.0786, L=512$, and stretched exponential

$(L=256)$. In the following, $\gamma / v$ is fixed to 1.9640 , which means $\eta=0.0360(5)$, which is close to the results of a compilation in Ref. [3]. This is in agreement with the recent estimate [26] of $\gamma / v=1.96390(45)$.

\section{Gaussian distribution?}

In Fig. 4, a deviation from the static critical behavior: $I(q) \propto q^{-\gamma / v}$ is observed in a region where $q<10 / L$. The finite lattice of our simulations modifies the scaling. Figures 1 and 2 show that, for $q=0$, the distribution of $A(q=0, j)$ (i.e., the total magnetization of the lattice) is not Gaussian, as is well known at a transition fixed point: $g_{0}^{(4)} \simeq 1.6$.

The distribution of $A(\vec{q}, j)$ is discussed from the study of $I(\vec{q}, j)$ for the various $|\vec{q}|$ and configurations $j$. Figure 5 plots the cumulants of the distributions of $I$ for different $|q|$ values with $\delta q=0.001$.

If $A(q)$ is Gaussian distributed, the distribution of the intensity $I(q)$ is exponential and the cumulants of its distribution are 1 (variance to mean ratio), 2 (skewness), and 6 (kurtosis). From Fig. 5, we observe that in the region where the $I(q)$ scaling is observed, the distribution of $A(q)$ is Gaussian. For $q>$ 0.2 , a deviation from the Gaussian distribution is observed, clearly connected to the lattice discretization. Figure 6 clearly shows that the Gaussian distribution is observed for $q>10 / L$ with an excellent precision for the two values of $L$ studied here.

\section{DYNAMICS}

\section{A. An example}

In Fig. 7 are plotted the $g^{(2)}(q, t)$ functions obtained in the $L=256$ system at $\beta=0.22165469$ from a set of 200 MCS, with a sample every MCS. From this figure, the main difficulties in the evaluations of $g^{(2)}(q, t)$ can be discussed:

(1) For small $q$ values, the estimates of the correlation functions are very rough because the timescale of the fluctuations is $\gg 200$ MCS and because the number of $\vec{q}$ in the $|\vec{q}|$ domain is small.

(2) For small $q$ values, Siegert's relation does not hold (non-Gaussian behavior).

(3) For small $q$ values, the observation of the detailed shape of $\gamma(q, t)$ vs $t$ is difficult.
For these reasons, the study focuses onto $q$ regions where the Gaussian behavior is observed. Independent time series of a limited number of MCS will be obtained by inserting some Wolff cluster updating between each time series.

\section{B. Testing a model}

To discuss the dynamics, the short-time behavior is first examined. In this case, the spin dynamics corresponds to a local change, and the collective aspects of the critical slowingdown should not be observed: In Eq. (7), this corresponds to $\mu=1$. In practice, the relaxation is in this case written with an exponential relaxation form:

$$
S(q, t)=S(q, t=0)-t / t_{0} \simeq S(q, 0) \exp [-t / \tau(q)],
$$

where $t_{0}$ corresponds to one spin flip $(\Delta m=2)$ and to the average flip probability in Eq. (11) $(\simeq 0.333): t_{0} \simeq 1.5 \mathrm{MCS}$. In the case $\mu=1, \tau(q) \propto S(q, 0) \propto q^{-\gamma / \nu}$. This short-time behavior was checked by introducing a stretched exponential

$$
g^{(2)}(q, t)=\exp \left\{-2\left[t /\left(\tau_{0} q^{-z}\right)\right]^{\mu}\right\} .
$$

In this equation, it is assumed that Eq. (10) is valid and that $\tau(q) \propto q^{-z}$ [see Eq. (5)]. The stretched exponential is an extension of the $t^{\gamma / \nu z}$ short-time behavior in Eq. (7).

In Fig. 8 are plotted a few results of a fit of simulations of the short time ( $t<10 \mathrm{MCS}, L=512$ ) correlations for a large $q$ domain (120 $q$ values from $q=0.078$ to $q=0.19$ ) with this equation. The fit to Eq. (17) in this large- $q$ domain gives excellent agreement: $z=1.971(4)$ and $\tau_{0}=0.169$.

This means that $\mu=\gamma / v z=0.996 \simeq 1$ : in this range, the dynamics is indiscernible from a classical exponential relaxation. In this case, combining Eqs. (17) and (14), one obtains $t_{0}=\tau_{0} / a_{1} \simeq 0.169 / 0.106=1.59$, close to previous estimate [Eq. (16)]. This checks that Eq. (16) is valid for short time.

One must here recall that only the difference between $\gamma / \nu$ and $z$ can characterize the anomalous slowing-down (see the discussion in Ref. [7]).

\section{The "long-term" behavior}

In Fig. 9, the results of various simulations are plotted for $L=512$. This figure only shows the region of $\gamma(q, t)$, 

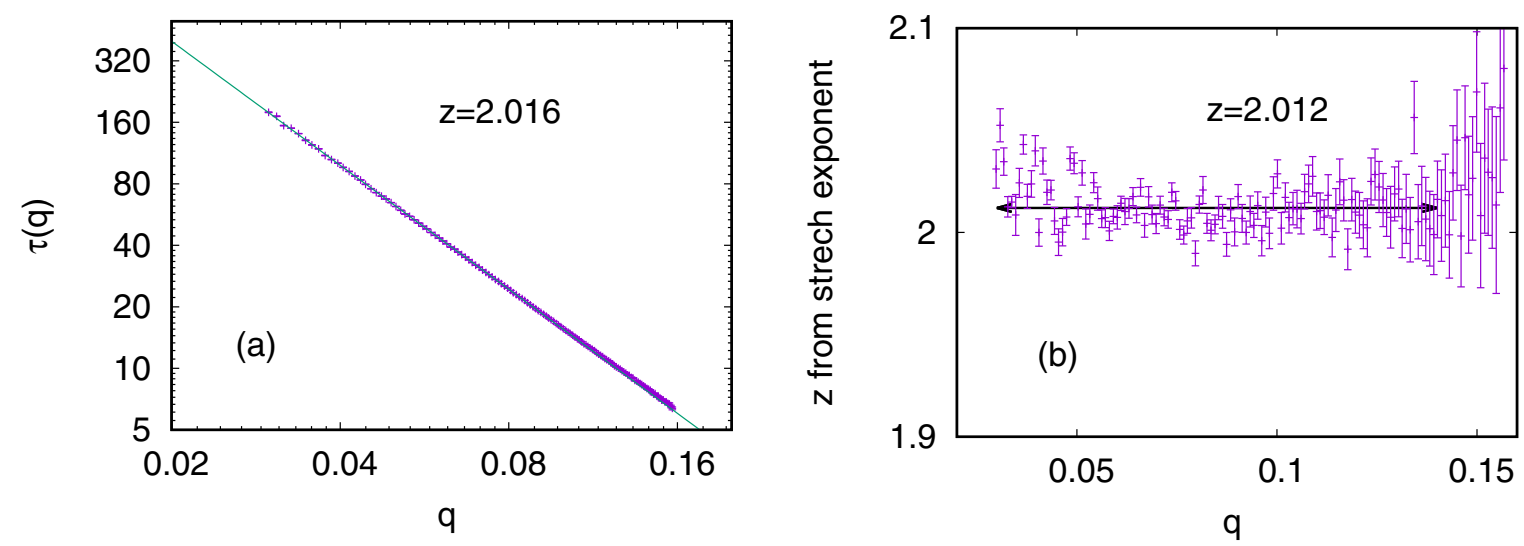

FIG. 11. In panel (a), $\tau(q) \propto q^{-2.016}$, in panel (b), $z \simeq 2.012$.

where $A(q)$ is Gaussian distributed. Different simulations with different time steps $(1,2,4,10,20,40 \mathrm{MCS})$ are plotted together.

The interpretation of the results makes use of the stretched exponential

$$
g^{(2)}(q, t)=\exp \left\{-2[t / \tau(q)]^{\mu}\right\} .
$$

Here, for each $q$ value, an estimate of $\tau(q)$ and of $z=\gamma / \nu \mu$ is obtained from the results at $t>10 \mathrm{MCS}$. A typical fit is shown in Fig. 10 for $q=0.0396$ and $q=0.0786$.

The resulting set of $\tau(q)$ is plotted in Fig. 11(a) and the various estimates of $z$ from the stretch exponent $\mu: z=\gamma / \nu \mu$ in Fig. 11(b).

\section{Unbiased estimates}

The estimated errors of the results of Fig. 10 are subjected to strong bias because of significant correlations between nearby MCS in the same series. To obtain unbias results, the values of $g^{(2)}(q, t)$ have been calculated independently. In this case, the correlations are calculated for each MCS time interval $\Delta t$. An example of the results is given in Fig. 12 for a

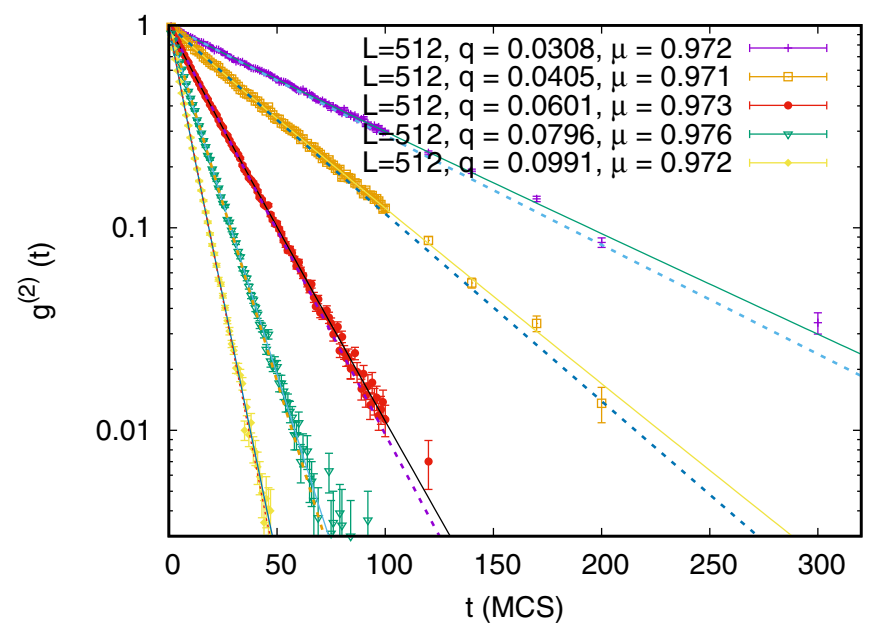

FIG. 12. $g^{(2)}(q, t)$ for $L=512$ and some $q$ values. The curves correspond to fits with Eq. (18), with an excellent agreement. The short-time results of Eq. (18) have also been plotted (dotted lines). set of $q$ vectors. All the points for each $q$ value and each time are statistically independent. This provides statistical tests of the stretched exponential hypothesis [Eq. (18)]. All fits in this figure show that this equation is valid for the full set of the values plotted in the figure. The exponent $\mu \simeq 0.975$ is clearly smaller than unity, although the curves in Fig. 12 do not differ too much from straight lines. In this figure are also plotted the short-time fit results, which are almost exponential.

In Fig. 13 are plotted all the results of the fits to Eq. (18): a plot of $q^{2} \tau(q)$ provides an estimate of $z=2.0150(18)$ [Fig. 13(a)] and $z=2.0145(6)$ [Fig. 13(b)] from the values of $\mu$. In this figure, the regions of $q$ where the scaling of the dynamics is observed seems to be limited to $0.03<q<0.08$.

\section{DISCUSSION}

The Gaussian shape of the distribution of $A(q)$ for intermediate $q$ values $(10 / L<q<0.2)$ is a characteristic property of the critical fluctuations in the fully critical regime. This can be connected to the Fortuin-Kasteleyn percolation model of the Ising transition [27]. In this case, the configuration of the moments can be described by partitioning the lattice in clusters, and it has been shown from a reformulation of the partition function that, for each cluster configuration, the spin sign of the clusters can be arbitrarily chosen. This is the base of the Swendsen-Wang algorithm [28,29].

These clusters have been studied in detail for the $2 \mathrm{~d}$ Ising system in Ref. [30], and the cluster distribution at $T_{c}$ for finite lattice can be roughly described with an "infinite" cluster which is percolating across the lattice, of total moment $\left|m_{\infty}\right| \propto L^{(5-\eta) / 2}$ and a distribution of a large number of smaller "finite" clusters. The distribution of Fig. 1 with two maxima corresponds to the dominant contribution of $m_{\infty}$ to $A(q=0)$ and, for intermediate- $q$ values, $A(q)$ corresponds to adding the amplitude scattered by finite clusters. As the sign of the moments can be arbitrarily changed, the central limit theorem holds, leading to a Gaussian distribution.

These remarks explain that carrying out MC simulations and analyzing the results of the dynamics from the study of $A(q=0, t)$ at $T_{c}$ like in Ref. [13] makes the observation of the critical slowing-down difficult: the scale invariance of the fluctuations is broken by the finite size $L$. This can also be 

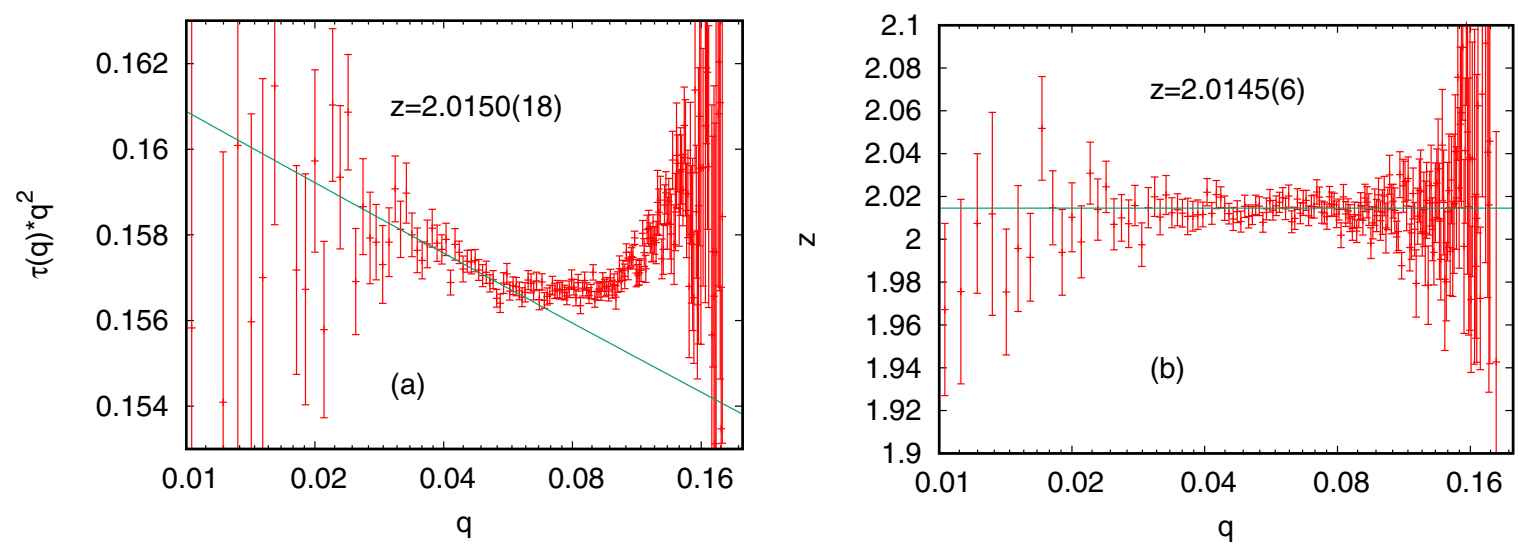

FIG. 13. In panel (a), $\tau(q) \propto q^{-2.015}$, in panel (b), $z \simeq 2.0145$ from estimates of $\mu=\gamma / \nu z$.

seen in Refs. [10] and [12], where the results were interpreted by two exponential behaviors.

This latter remark is connected to the observation of stretched exponentials, introduced from Eq. (7). The time domain where a nonlinear time behavior is valid can be extended: in Eq. (7), only the condition $t / t_{0} \ll \xi^{z}$, which corresponds here to $t / t_{0} \ll L^{z}$, has to be fulfilled. This paper proposes that the correlation functions are essentially described by stretched exponentials, even for $t / t_{0}>q^{-z}$, provided that $q \gg \xi^{-1}$ (or $q \gg L^{-1}$ ).

The estimate of $z=2.0145$ given here is close to the results of dynamic renormalization group results $(z=2.017)$ [9], significantly different from Ref. [5], where $\tau$ is obtained by two methods: evaluation of $\tau=\sum g^{(1)}(q, t)$ and, assuming a simple exponential, estimating $\tau(q)=-|t| / \ln \left[g^{(1)}(q, t)\right]$ for large $t$. In the first method, $g^{(1)}(q, t)$ is sensitive to size and short-time biases and in the second method, the observation of an exponential is extremely difficult because the large $t$ means $t / t_{0} \gg \xi^{z}\left(\right.$ or $\left.t / t_{0} \gg L^{z}\right)$.
The results of the simulations carried out in this paper can have important consequences: the Ising model corresponds to a $\phi^{4}$ model of criticality, with a one-dimensional order parameter. MC Ising introduces an UV cutoff (the lattice constant $a$ ) and an IR cutoff $(L a)$. The small difference observed in Fig. 12 between an exponential and a stretched exponential is extended for a fully critical system to an infinite number of time orders of magnitude. In this case, the fact that $\mu-1=-0.025$ qualitatively changes the dynamics, but its observation in an XPCS experiment can be difficult.

\section{ACKNOWLEDGMENTS}

The results of the set of independent correlations were carried out on the SIMaP multiprocessor computer, and the author wants to thank R. Poloni for having opened this machine. Fruitful discussions with M. Verdier, A. Deschamps, and $\mathrm{G}$. Beutier helped the presentation of this paper.
[1] P. C. Hohenberg and B. I. Halperin, Rev. Mod. Phys. 49, 435 (1977).

[2] B. I. Halperin and P. C. Hohenberg, Phys. Rev. 177, 952 (1969).

[3] J. C. L. Guillou and J. Zinn-Justin, J. Phys. (Paris) 48, 19 (1987).

[4] R. Folk and G. Moser, J. Phys. A: Math. Gen. 39, R207 (2006).

[5] P. Calabrese, V. Martín-Mayor, A. Pelissetto, and E. Vicari, Phys. Rev. E 68, 016110 (2003).

[6] J. C. Anglesd'Auriac, R. Maynard, and R. Rammal, J. Stat. Phys. 28, 307 (1982).

[7] C. De Dominicis, E. Brézin, and J. Zinn-Justin, Phys. Rev. B 12, 4945 (1975).

[8] H. Kleinert, J. Neu, V. Schulte-Frohli, K. Chetyrkin, and S. Larin, Phys. Lett. B 272, 39 (1991).

[9] V. V. Prudnikov, A. V. Ivanov, and A. A. Fedorenko, JETP Lett. 66, 835 (1997).

[10] S. Wansleben and D. P. Landau, J. Appl. Phys. 61, 3968 (1987).

[11] P. Grassberger, Phys. A (Amsterdam, Neth.) 214, 547 (1995).

[12] S. Wansleben and D. P. Landau, Phys. Rev. B 43, 6006 (1991).

[13] H. O. Heuer, J. Phys. A: Math. Gen. 25, L567 (1992).
[14] F. Livet, F. Bley, J.-P. Simon, R. Caudron, J. Mainville, M. Sutton, and D. Lebolloc'h, Phys. Rev. B 66, 134108 (2002).

[15] F. Livet, M. Fèvre, G. Beutier, F. Zontone, Y. Chushkin, and M. Sutton, Phys. Rev. B 98, 014202 (2018).

[16] F. Livet, Acta Crystallogr., Sect. A: Found. Crystallogr. 63, 87 (2007).

[17] E. Jakeman and P. N. Pusey, J. Phys. A: Math. Gen. 8, 369 (1975).

[18] P. Pusey and W. van Megen, Physica A (Amsterdam, Neth.) 157, 705 (1989).

[19] P. Raimondi, in Proceedings of International Particle Accelerator Conference (IPAC'16), Busan, Korea, International Particle Accelerator Conference (JACoW, Geneva, Switzerland, 2016), pp. 2023-2027.

[20] A. Jaster, J. Mainville, L. Schülke, and B. Zheng, J. Phys. A: Math. Gen. 32, 1395 (1999).

[21] U. Wolff, Phys. Rev. Lett. 62, 361 (1989).

[22] F. Livet, Europhys. Lett. 16, 139 (1991).

[23] K. Binder, Z. Phys. B: Condens. Matter Quanta 43, 119 (1981).

[24] F. J. Wegner, Phys. Rev. B 5, 4529 (1972). 
[25] P. Hou, S. Fang, J. Wang, H. Hu, and Y. Deng, Phys. Rev. E 99, 042150 (2019).

[26] A. M. Ferrenberg, J. Xu, and D. P. Landau, Phys. Rev. E 97, 043301 (2018).

[27] C. M. Fortuin and P. W. Kasteleyn, Physica 57, 536 (1972).
[28] R. H. Swendsen and J.-S. Wang, Phys. Rev. Lett. 58, 86 (1987).

[29] J.-S. Wang and R. H. Swensen, Phys. A (Amsterdam, Neth.) 167, 565 (1990).

[30] M. D. De Meo, D. W. Heermann, and K. Binder, J. Stat. Phys. 60, 585 (1990). 